\title{
Editorial: A Brief Perspective to the Development of Emerging Thermoelectric Materials
}

\author{
Zhenzhen Qin ${ }^{1}$, Hongbin Zhang ${ }^{2 *}$ and Guangzhao Qin $^{3 *}$ \\ ${ }^{1}$ International Laboratory for Quantum Functional Materials of Henan, School of Physics and Microelectronics, Zhengzhou \\ University, Zhengzhou, China, ${ }^{2}$ Institute of Materials Science, Technische Universität Darmstadt, Darmstadt, Germany, ${ }^{3}$ State \\ Key Laboratory of Advanced Design and Manufacturing for Vehicle Body, College of Mechanical and Vehicle Engineering, Hunan \\ University, Changsha, China
}

Keywords: thermoelectricity, thermal transport, optimization, SPIN, high-through computation

\section{Editorial on the Research Topic}

\section{A Brief Perspective to the Development of Emerging Thermoelectric Materials}

\section{OPEN ACCESS}

Edited by:

Hanfeng Liang,

Xiamen University, China

Reviewed by:

Xiangming Xu,

King Abdullah University of Science and Technology, Saudi Arabia

Qiu Jiang,

University of Electronic Science and

Technology of China, China

*Correspondence:

Hongbin Zhang

hongbin.zhang@tu-darmstadt.de

Guangzhao Qin

qin.phys@gmail.com

Specialty section:

This article was submitted to

Energy Materials,

a section of the journal

Frontiers in Materials

Received: 28 October 2021 Accepted: 09 February 2022

Published: 28 February 2022

Citation:

Qin Z, Zhang H and Qin G (2022) Editorial: A Brief Perspective to the

Development of Emerging

Thermoelectric Materials.

Front. Mater. 9:803853.

doi: $10.3389 /$ fmats.2022.803853
The ever-growing energy issue brings forth a strong impetus for efficient harvesting and conversion of heat into electrical energy, where thermoelectric (TE) materials play a pivotal role. Such materials provide a simple and environmentally friendly solution for the thermoelectric conversion, applicable for automobiles and high-power electrical appliances which produce lots of waste heat.

The development of high-performance TE materials and their optimization have attracted intensive attention in the materials science and energy communities, with one of the main goals to boost the TE figure of merit $Z T=\frac{S^{2} \sigma}{\kappa} T=\frac{S^{2} \sigma}{\kappa_{e}+\kappa_{l}} T$, where $S$ is the Seebeck coefficient, $\sigma$ is the electrical conductivity, $\kappa_{e}$ is the electronic thermal conductivity, $\kappa_{l}$ is the lattice thermal conductivity, and $T$ is the absolute temperature. In this regard, ZT can be efficiently enhanced by lowering the thermal conductivity, which can be achieved by lots of possible manners, such as designing the geometry structures at the micro/nanoscales. For instance, the strain-modulated phonon transport behavior of the monolayer $\mathrm{H}$-decorated pentagonal silicene (penta-SiH) and bilayer pentagonal silicene (penta-Si), and one hexagonal H-decorated silicene structures (H-silicene) are comparably studied from first-principles (Liu et al.). For both pentagonal silicene structures, the thermal conductivity first shows a large improvement (90\% for monolayer penta-SiH and $400 \%$ for bilayer penta-Si) as tensile strain increases from 0 to $10 \%$ and then stabilizes with a strain larger than $10 \%$. A detailed analysis shows that the phonon group velocity and phonon lifetime of both structures increase with applied strain, and the phonon lifetime plays the major role in the improvement of thermal conductivity. Furthermore, based on the detailed analysis between the pentagonal (penta-SiH) and hexagonal silicene structures (H-silicene), the difference in out-of-plane phonon scattering cannot be ignored. Furthermore, the thermal conductivities of $\mathrm{Mg}$ - and Si-doped $\mathrm{GaN}$ are studied based on the first-principles method combined with the solvent of phonon Boltzmann transport equation (BTE) ( $\mathrm{Li}$ et al.). Li et al. found that both the thermal conductivities of $\mathrm{Mg}$ - and Si-doped $\mathrm{GaN}$ reach the ultra-low values, and the thermal conductivity of Si-doped $\mathrm{GaN}$ is even an order of magnitude lower than that of $\mathrm{Mg}$ doped GaN.

In recent years, two-dimensional (2D) materials have been extensively investigated for fabricating miniaturized electronic devices, where the thermal management and thermoelectric conversion become essential. Interestingly, an increasing number of $2 \mathrm{D}$ materials have been predicted to have the potential applications in TE via high-throughput calculations 


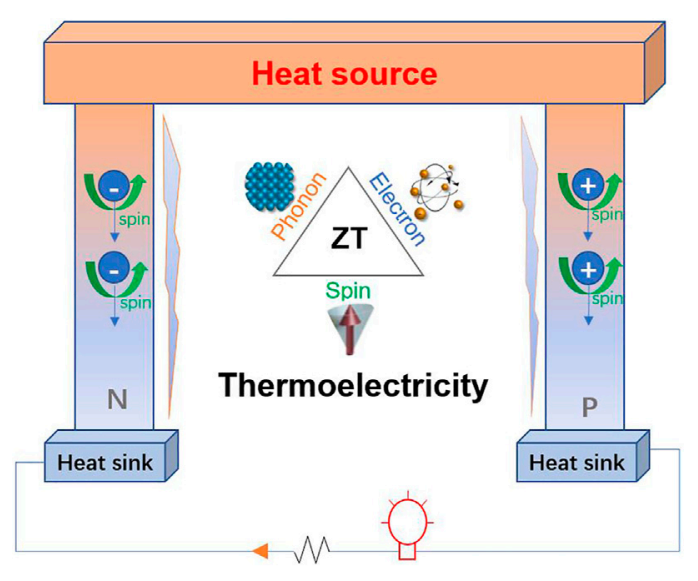

FIGURE 1 | Schematic diagram of thermoelectricity.

(Jia et al., 2020; Sarikurt et al., 2020). Electronic fitness function as a new descriptor to estimate TE performance of semiconductors is usually used for screening novel TE materials. Among them, the monolayer $\mathrm{GeS}_{2}$ has been recently confirmed as a good candidate for $2 \mathrm{D}$ materials with weak interlayer coupling from theory and experiment study (Yan et al., 2022). In this topic, Wang et al. systematically investigated the TE performance of monolayer $\mathrm{GeS}_{2}$, including both the electronic and the thermal transport properties (Wang et al.). As compared with other typical TE monolayer materials, the monolayer

\section{REFERENCES}

Jia, T., Feng, Z., Guo, S., Zhang, X., and Zhang, Y. (2020). Screening Promising Thermoelectric Materials in Binary Chalcogenides Through High-Throughput Computations. ACS Appl. Mater. Inter. 12, 11852-11864. doi:10.1021/acsami. $9 \mathrm{~b} 23297$

Sarikurt, S., Kocabaş, T., and Sevik, C. (2020). High-Throughput Computational Screening of 2D Materials for Thermoelectrics. J. Mater. Chem. A 8 (37), 19674-19683. doi:10.1039/D0TA04945J

Yan, H.-J., Li, Z., Liu, S.-C., Wang, X., Zhang, X., Xue, D.-J., et al. (2022). Investigation of Weak Interlayer Coupling in 2D Layered GeS2 from Theory to Experiment. Nano Res. 15 (2), 1013-1019. doi:10.1007/s12274021-3589-3
$\mathrm{GeS}_{2}$ exhibits excellent electronic transport properties but a relatively high lattice thermal conductivity of 5 . $71 \mathrm{~W} \mathrm{~m}^{-1} \mathrm{~K}^{-1}$ at $500 \mathrm{~K}$. Thus, an unsatisfactory $Z T$ value of 0.23 is reached. Such a low $Z T$ value indicates that beyond the electronic transport properties, it is necessary to also consider the thermal transport properties through high-throughput calculations to screen the thermoelectric materials with excellent performance.

This collection on the investigation of TE materials or the closely related thermal conductivity provides a comprehensive perspective, which is expected to stimulate research inspiration in the TE field and advance the fundamental understanding of TE performance. Due to the role of electrons, spins, and the lattice vibrations (phonon) and their couplings have a significant effect on the thermal transport and thermoelectric performance (Figure 1), more novel strategies are expected to boost TE performance in future, such as spin modulation, etc. Notably, the Rashba effect modulation is expected to optimize the electronic and the thermal transport properties simultaneously. In addition, for searching high performance TE materials through high-throughput calculations and machine learning techniques, new descriptors are also deserved to be explored, such as by constructing from existing descriptors of bandgap, effective mass, etc.

\section{AUTHOR CONTRIBUTIONS}

All authors listed have made a substantial, direct, and intellectual contribution to the work and approved it for publication.

Conflict of Interest: The authors declare that the research was conducted in the absence of any commercial or financial relationships that could be construed as a potential conflict of interest.

Publisher's Note: All claims expressed in this article are solely those of the authors and do not necessarily represent those of their affiliated organizations, or those of the publisher, the editors and the reviewers. Any product that may be evaluated in this article, or claim that may be made by its manufacturer, is not guaranteed or endorsed by the publisher.

Copyright (c) 2022 Qin, Zhang and Qin. This is an open-access article distributed under the terms of the Creative Commons Attribution License (CC BY). The use, distribution or reproduction in other forums is permitted, provided the original author(s) and the copyright owner(s) are credited and that the original publication in this journal is cited, in accordance with accepted academic practice. No use, distribution or reproduction is permitted which does not comply with these terms. 